\title{
A Neural Network Based Technique to Locate and Classify Microcalcifications in Digital Mammograms
}

\author{
Brijesh Verma \\ School of Information Technology, Griffith University, Gold Coast Campus, Qld 9726, Australia \\ E-mail: B.Verma@gu.edu.au
}

\begin{abstract}
This paper proposes a technique that extracts suspicious areas containing microcalcifications in digital mammograms and classifies them into two categories whether they contain benign or malignant clusters. The centroids and radiuses provided by expert radiologist are being used to locate and extract suspicious areas. Neural network's generalisation abilities are used to classify them into benign or malignant. The technique has been implemented in $\mathrm{C}++$ on the SP2 supercomputer. The database from the Department of Radiology at the University of Nijmegen and Lawrence Livermore National Laboratory has been used for the experiments. The preliminary results are very promising. Some of them are presented in this paper.
\end{abstract}

Keywords: Neural Networks, Image Processing, Digital Mammography, Medical Applications, Breast Cancer, Microcalcifications, Classification Techniques

\section{Introduction}

Breast cancer is a leading cause of fatality in women, with approximately 1 in 12 women affected by the disease during their lifetime $[1,2]$. In Australia, approximately one in 13 women develop breast cancer at sometime in their life, and about 1 in 25 Australian women [2] die because of breast cancer.

Digital mammography currently offers the best cancer control strategy for early detection of breast cancer. A number of research projects [3, 4] [7-16] have been directed towards the development of more efficient methods for detecting and treating breast cancer. Most of the work on computer analysis of mammograms has concentrated on detection of small abnormalities, specifically microcalcifications $[3,4][7-16]$. This can be a difficult, tedious, and time-consuming task since mammographic images are very complicated, big and poor in contrast. The segmentation process for extraction of such regions is not reliable and accurate. There are a few researchers $[3,4,10,13]$ all over the world who tried to combine neural networks and detection of microcalcifications. Some of them fed the full mammographic images to the neural network, others tried to divide mammograms into small windows and some tried to extract features $[10,14]$. Research showed that the last approach is the best one, however the reported results for the classification of microcalcifications in the literature are not very impressive and need to be improved.

The objective of this research is to overcome the above-mentioned problems by combining a technique to locate the areas containing the microcalcifications and learning and generalisation abilities of the feedforward neural network.

The proposed approach is based on the previous work conducted by the author. In [4], he proposed a technique to reduce the size of mammograms by finding the nipple position. The technique is used to reduce the size of mammograms and focus only on suspicious areas. Also, in [3], the author proposed a neural network technique for detection of microcalcifications in digital mammography. We obtained interesting results but not as we expected and we found that it was because of large areas fed to the neural network for training. Therefore, in this research we used expert radiologists experience and developed a technique to locate the suspicious areas and fed small areas containing microcalcifications to the neural network.

The remainder of this paper is organised as follows: In Section 2, we describe the proposed technique. Some experimental results are shown in Section 3. The comparison and discussion of results is presented in Section 4. Section 5 discusses future research. Finally, Section 6 concludes the paper. 


\section{Proposed techniques}

In this section, we describe the proposed technique and steps required to process a database of mammograms.

We downloaded a database from the Department of Radiology at the University of Nijmegen and also we have purchased a database through our research grant from Lawrence Livermore National Laboratory. All images (mammograms) were in raw format and were of size $2048 \times 2048$. They use 12 bits ( 2 bytes) per pixel of grey-level information which were recorded with a Kodak MIN-R/S0177 screen film combination using various types of equipment. Some samples of mammographic images containing microcalcifications (benign/malignant) are shown below in Figure 1.

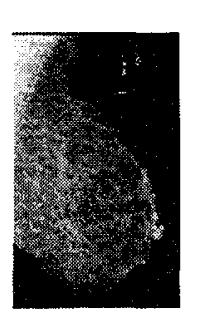

(a)

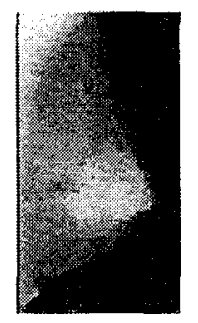

(b)

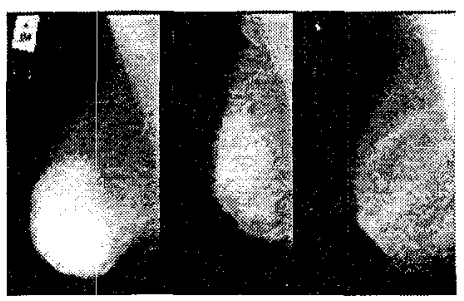

(c)
a. microcalcifications
b. architecture distortion
c. comprehensive set of normal

\section{Figure 1. Some samples of mammograms}

We have two files, the first with raw images and a second file with the centre position, radiuses and the number of microcalcification clusters which were marked by two expert radiologists. We read in the centre position and radius, our algorithm allocated the cluster on the mammograms and fitted it into a quadratic window which was used by the neural network.

\subsection{Classification Technique for Extracted Areas}

A feedforward neural network with a single hidden layer is used to classify extracted areas containing microcalcifications into benign or malignant clusters. A full diagram of a classification system is shown below in Figure 2. As shown in the Figure, the areas with microcalcifications detected by two expert radiologists, located and extracted by the proposed technique, are taken for training of the neural network. After training, the areas selected by the users from new mammograms can be classified into benign or malignant.

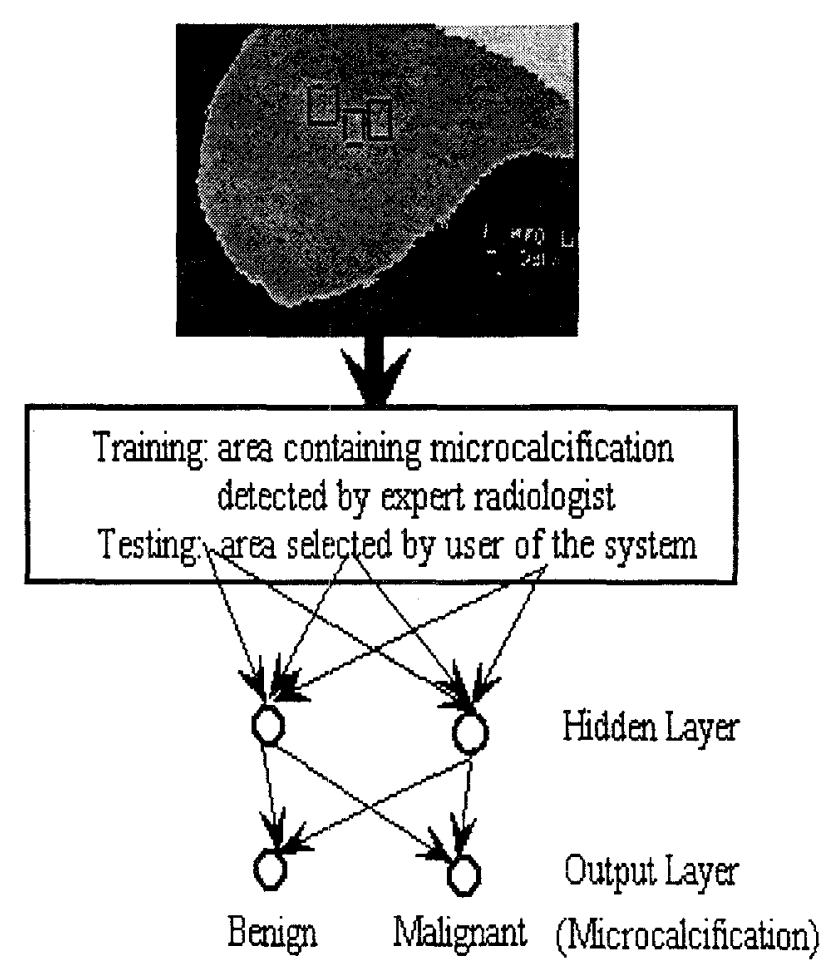

Figure 2. Proposed classification system

\subsubsection{Neural Network Training Algorithms}

In this research, we investigate two training algorithms. An error backpropagation (EBP) $[4,5]$ with momentum and direct solution method (DSM) [6] based training algorithms were employed to train a feedforward neural network.

EBP is an iterative method and DSM is a noniterative method. The advantage of the latter one is fast convergence. Both algorithms were compared in relation to training time and overall classification accuracies.

\section{Experimental Results}

The proposed approach has been implemented in C on the SP2 supercomputer. A SUN Sparc workstation and a Supercomputer (IBM SP2) are being used for computing and training the neural networks. A database $[11,12]$ of 21 mammographic images from 21 patients 
have been used. Currently, we are testing our technique on the database provided by the Lawrence Livermore National Laboratory.

We have implemented and used two algorithms, one for converting raw images (mammograms) into a pixel image and second to locate and extract the suspicious areas based on centroid and radiuses marked by expert radiologists. We extracted 105 areas containing 76 malignant and 29 benign microcalcifications. We used 90 for training and 15 for testing. We conducted many experiments using both (EBP and DSM) algorithms, varying the number of hidden units, learning rate, momentum and the number of iterations. The results for both algorithms are shown below in Tables 1 and 2 respectively.

\section{Table 1: Classification using EBP}

$$
\begin{array}{ll}
\text { \# of inputs } & =1257 \\
\text { \# of outputs } & =1 \\
\text { \# of hidden layers } & =1 \\
\text { \# of training pairs } & =90 \\
\text { \# of test pairs } & =15
\end{array}
$$

\begin{tabular}{|c|c|c|c|c|c|c|}
\hline 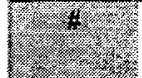 & $\begin{array}{l}\text { \# Hidden } \\
\text { Units }\end{array}$ & Hry & $\alpha$ & 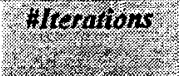 & \multicolumn{2}{|c|}{$\begin{array}{c}\text { Recognition } \\
\text { Rate [\%] }\end{array}$} \\
\hline 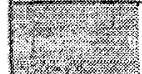 & & & & $\left(\frac{1}{2}\right)$ & $\begin{array}{l}\text { Training } \\
\text { Set }\end{array}$ & $\begin{array}{l}\text { Test } \\
\text { Set }\end{array}$ \\
\hline 1 & 10 & 02 & 0.4 & 1000 & 97.8 & 86.6 \\
\hline 2 & 16 & 131 & 0.1 & 101 & 100 & 86.6 \\
\hline the & 16 & 0,3 & 0.5 & 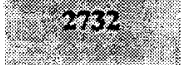 & 100 & 86.6 \\
\hline
\end{tabular}

\begin{tabular}{|c|c|c|c|c|}
\hline 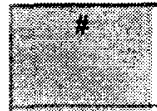 & $\begin{array}{c}\# \\
\text { Hidden } \\
\text { Units } \\
\end{array}$ & Therations & \multicolumn{2}{|c|}{ Recognition Rate [\%] } \\
\hline lint? & & & $\begin{array}{l}\text { Training } \\
\text { Set }\end{array}$ & Test Set \\
\hline $1+v$ & 10 & 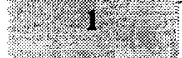 & 100 & 56 \\
\hline 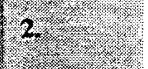 & 16 & & 100 & 77 \\
\hline 3. & 35 & P. & 100 & 86.6 \\
\hline
\end{tabular}

Table 2: Classification using DSM

$$
\begin{array}{ll}
\text { \# of inputs } & =1257 \\
\text { \# of outputs } & =1 \\
\text { \# of hidden layers } & =1 \\
\text { \# of training pairs } & =90 \\
\text { \# of test pairs } & =15
\end{array}
$$

\section{Comparison and Discussion of Results}

Comparing results with other researchers is one of the most difficult tasks because of different techniques, parameters, data, etc, used by other researchers. The most similar environment to my technique to classify microcalcifications in Digital Mammography, was proposed by Zheng in [10], where he presented a multistage neural network based technique, in which he claimed recognition rates of $81 \%$. We obtained an overall recognition rate of $50 \%$ in our previous research [3], in which we tried to feed full images (mammograms) to the neural network. As can be seen in Table 1 , we received overall recognition rate of $87 \%$ for both methods, which is much higher than Zheng's as well as our previous results. We conducted many experiments varying hidden units and with different sets of testing data but we could not get better recognition rates. DSM was much faster in training and more accurate than EBP with the use of more hidden units. We think that the overall classification rate could be improved with a larger training set.

\section{Future Research}

We would like to test our technique with a larger database, which we have already started with a database obtained from the Lawrence Livermore National Laboratory.

Also, we would like to improve our technique by using an unsupervised Kohonen neural network which can extract features from extracted areas then feed those features to another feedforward neural network.

\section{Conclusions}

In this paper, we have described a technique to locate and classify microcalcifications in digital mammography. The proposed method can be used as a second opinion and provides help to radiologists. It reduces time and improves accuracy rates for detection and classification of microcalcifications. We applied the proposed approach to 21 mammograms and extracted suspicious areas containing benign and malignant calcifications. We trained a neural network with 90 microcalcifications and tested it with 15 , as can be seen in Tables 1 and 2 , the results are very promising. This research project is ongoing and we expect even better results using a larger database.

\section{Acknowledgment}

This research work was supported by Griffith University Research Grant No. TE CI 2888043.

\section{References}

[1] The Mammographic Image Analysis Society (MIAS), UK. 
[2] National Breast Cancer Centre, Sydney, Australia

[3] B.K. Verma, An automatic method for detection and classification of microcalcifications using AINs, Proceedings of Fourth IASTED International Conference on Robotics and Manufacturing, IASTED RM'96, pp. 84-88, Hawaii, USA, 1996.

[4] B.K. Verma, A neural network for detection of boundary and nipple position in digital mammography, Proceedings of the International Conference on Intelligent And Cognitive Systems, ICICS'96, pp. 200-205, Tehran, Iran, 1996.

[5] B.K. Verma, Recognition of rotating images using an automatic feature extraction technique and neural networks, International Journal of Neural Systems (INS), vol. 8, no. 2, pp. 201-208, 1997.

[6] B.K. Verma, Fast Training of Multilayer Perceptrons(MLPs). IEEE Transactions on Neural Networks, vol. 8, no. 6, pp. 1-8, USA, 1997.

[7] J. Parker and D.R. Dance, Digital Mammography: Image Analysis and Automatic Classification of Calcification in Ductal Carcinoma in Situ, State of the Art in Digital Mammographic Image Analysis, World Scientific Publishing, Co., 1994.

[8] Y. Chitre, A.P. Dhawan, ANN Based Classification of Mammographic Microcalcifications using Image Structure Features, State of the Art in Digital Mammographic Image Analysis, World Scientific Publishing, Co., 1994.

[9] S. Hanjal and P. Taylor, Classifying Mammograms by Detecting: Soting for Screening, State of the Art in Digital Mammographic Image Analysis, World Scientific Publishing, Co., 1994.

[10] B. Zheng and W. Qian, Multistage NN for Pattern Recognitionin Mammogram Screening, Proceedings of IEEE International Conf. on Neural networks, ICNN"94, Orlando, USA, 1994.

[11] N. Karssemeijer, Recognition of Clustered Microcalsifications Using a Random Feild Model, Proceedings of Biomedical Irnage Processing, IPMI"93, 1994.

[12] N. Karssemeijer, Adaptive Noise Equalization and Image Analysis in Mammography, Proceedings Information Processing in Medical Imaging, IPMI"93, 1993.

[13] K. S. Woods and C. C. Doss, Comparative Evaluation of Pattern Recognition Techniques for Detection of Microcalcifications in Mammography, International Journal of Pattern Recognition and Artificial Intelligence, 1993.

[14] J. L. Solka and W. L. Postan, The Detection of Micro-Calcifications in Mammograpic Images Using High Dimentional Features, Proceedings of the IEEE Seventh Symposium on ComputerBased Medical Systems, 1994.

[15] K.S. Woods and C.C Doss, Comparative Evaluation of Pattern Recognition Techniques for Detection of Microcalcifications in Mammography, International Journal of Pattern Recognition and Artificial Intelligence, pp. 8085, 1993.

[16] C.E. Priebe and J.L. Solka, R.A. Lorey, G.W. Rogers, L.P. Clarke and R.A. Clark, The Application of Fractal Analysis to Mammographic Tissue Classification, Cancer Letters 77, pp. 183-189, 1994.

[17] W. Zhang, Computerized Detection of Clustered Microcalcifications in. Digital Mammograms using a Shift-invariant Artificial Neural Networks, Med. Phys. $20: 881,1993$.

[18] Y. Wu, Computerized Detection of Clustered Microcalcifications in Digital Mammograms: Applications of Artificial Neural Networks, Med. Phys. 19 (3), May, 1992. 